\title{
Associação entre autoavaliação de saúde e tipos de atividades de vida diária em idosos
}

\section{Association between health self-assessment and types of activities of daily living in older adults}

\author{
Joyce Souza Lemes (D), Valéria Pagotto² (D), Priscielle Karla Alves Rodrigues (D), Ivânia Vera ${ }^{3}$ (D), \\ Erika Aparecida Silveira' (D) \\ 'Universidade Federal de Goiás (UFG) - Goiânia (GO), Brasil. \\ ${ }^{2}$ Faculdade de Enfermagem, Universidade Federal de Goiás (UFG) - Goiânia (GO), Brasil. \\ ${ }^{3}$ Universidade Federal de Goiás (UFG) - Catalão (GO), Brasil.
}

Como citar: Lemes JS, Pagotto V, Rodrigues PKA, Vera I, Silveira EA. Associação entre autoavaliação de saúde e tipos de atividades de vida diária em idosos. Cad Saúde Colet, 2021;29(2):251-259. https://doi.org/10.1590/1414-462X202129020450

\section{Resumo}

Introdução: Apesar das evidências de associação entre incapacidade funcional e autoavaliação de saúde ruim, ainda existe uma lacuna na literatura acerca da influência do tipo de atividade de vida diária na saúde dos idosos. Objetivo: Identificar a prevalência de autoavaliação de saúde ruim de idosos e sua associação com a dependência para cada tipo de atividade da vida diária. Método: Estudo transversal de base populacional, com amostra probabilística de 890 idosos residentes em Goiânia. Avaliaram-se variáveis demográficas, internações, número de morbidades, atividades básicas e instrumentais da vida diária. Realizou-se uma análise múltipla hierarquizada, considerando como medida de efeito a razão de prevalência. Resultados: A prevalência de autoavaliação de saúde ruim foi de $11,5 \%$. Os fatores associados foram: ter três ou mais doenças (RP: 4,26; $95 \%$ IC: 1,89-9,62), internação no último ano (PR: 1,59; 95\% IC: 1,10-2,28), e incapacidades para:Transferir-se (RP: 2,60; 95\% IC: 1,30-8,45), fazer compras (RP: 1,98; 95\% IC: 1,16-3,39), usar transporte (RP: 3,86; $95 \%$ IC: 1,41-10,5), e usar telefone (RP: 1,60; $95 \%$ IC: 1,00-2,63). Conclusão: A relação entre incapacidade e percepção negativa de saúde sinaliza a importância de incluílas na avaliação de idosos em diferentes contextos da atenção à saúde, uma vez que a dependência pode refletir em alterações nas condições reais de saúde e qualidade de vida dos idosos. Palavras-chave: atividades cotidianas; autoavaliação de saúde; idoso; multimorbidade.

\begin{abstract}
Background: Despite the evidence on the association between functional disability and poor self-rated health, there is still a gap on the influence of the type of activity of daily living (ADL) on the health of the elderly population. Objective: To identify the negative self-assessment of health by older adults and its association with dependence on each type of ADL. Method: A cross-sectional, population-based study with probabilistic data conducted with 890 elderly residents in Goiânia, state of Goiás, Brazil. The following variables were assessed: sample demography, hospitalization, number of morbidities, and basic and instrumental ADLs. A hierarchical multiple analysis was performed considering prevalence ratio (PR) as measure of effect. Results: The prevalence of poor self-rated health was $11.5 \%$. Associated factors were as follows: multimorbidity (PR: $4.26 ; 95 \% \mathrm{Cl} 1.89-9.62)$, hospitalization in the past year (PR: 1.59; $95 \% \mathrm{Cl} 1.10-2.28)$, and disability for transferring (PR: $2.60 ; 95 \% \mathrm{Cl} 1.30-8.45)$, shopping (PR: $1.98 ; 95 \%$ $\mathrm{Cl}$ 1.16-3.39), use of transportation (PR: 3.86; 95\% Cl 1.41-10.5) and use of the telephone (PR: 1.60; 95\% $\mathrm{Cl}$ 1.00-2.63). Conclusion: The association between functional disability and negative self-rated health indicate the importance of including them in the evaluation of different health care modalities, since dependence may reflect changes in the actual health conditions and quality of life of older adults.
\end{abstract}

Keywords: activities of daily living; self-assessment; older adults; multimorbidity.

Este é um artigo publicado em acesso aberto (Open Access) sob a licença Creative Commons Attribution, que permite uso, distribuição e reprodução em qualquer meio, sem restrições desde que o trabalho original seja corretamente citado. 


\section{INTRODUÇÃO}

A autoavaliação de saúdeéum indicador amplamente utilizado em pesquisas epidemiológicas $\operatorname{com~idosos}^{1-3}$ por sua propriedade em predizer a morbidade e subsequente mortalidade ${ }^{4}$. No Brasil, dados do Projeto Bambuí mostraram que a autoavaliação negativa de saúde possui capacidade de prever a mortalidade mesmo após uma década de seguimento dos idosos ${ }^{4}$.

A prevalência de autoavaliação negativa da saúde em idosos varia de $12,6 \%$ a $51,9 \%$ em diferentes regiões brasileiras ${ }^{5}$. Além das desigualdades sociais ${ }^{2}$, as múltiplas morbidades ${ }^{6}$, as quedas ${ }^{3}$ e a incapacidade ${ }^{7}$ apresentaram associação com a autoavaliação de saúde em estudos prévios ${ }^{3,6,7}$.

Assim como a autoavaliação da saúde, a capacidade funcional também é um indicador muito utilizado em pesquisas com idosos, pois expressa sua dependência para desempenhar atividades de vida diária (AVDs), condição indispensável para a autonomia e a manutenção da qualidade de vida ${ }^{8,9}$. Estudos epidemiológicos demonstram que a dependência tanto para atividades básicas de vida diária (ABVDs) como para as atividades instrumentais de vida diária (AIVDs) podem impactar negativamente na saúde ${ }^{10-12}$, influenciando a percepção sobre sua condição geral de saúde.

Alguns estudos mostraram que a incapacidade funcional está associada à pior autoavaliação do estado de saúde dos idosos ${ }^{13-15}$. Em estudo de seguimento, a pior percepção de saúde foi um fator preditor independente para o declínio nas AIVDs ${ }^{14}$. Entretanto, não foram encontrados estudos analisando a relação entre cada tipo de AVD. Sabe-se que a dependência parcial ou total em alguma das atividades sinaliza problemas de saúde muitas vezes ocultos ou subdiagnosticados.

Diante disso, embora existam evidências de que a incapacidade funcional está associada à autopercepção de saúde ruim ${ }^{13-15}$, analisar o tipo de atividade diária que tem maior associação pode contribuir para o melhor acompanhamento e intervenções precoces nos idosos. Sendo assim, o objetivo deste estudo foi estimar a prevalência de autoavaliação de saúde negativa conforme as condições sociodemográficas, de saúde e tipos de ABVDs e AIVDs de idosos que vivem na comunidade.

\section{MÉTODO}

Este estudo está inserido na pesquisa matriz intitulada "Situação de saúde da população idosa do município de Goiânia" e foi aprovado pelo Comitê de Ética em Pesquisa da Universidade Federal de Goiás (Protocolo n 050/2009). Integra a Rede de Vigilância à Saúde do Idoso no Estado de Goiás (REVISI) e foi financiado pela Fundação de Amparo à Pesquisa do Estado de Goiás (FAPEG).

Trata-se de um estudo transversal, de base populacional desenvolvido em Goiânia, capital do estado de Goiás, atualmente com uma população de 1.495 .705 de pessoas ${ }^{16}$. À semelhança do Brasil e da maioria das capitais brasileiras, Goiânia também passa por um processo de envelhecimento populacional.

Os detalhes metodológicos foram descritos em publicação prévia ${ }^{17}$. Resumidamente, o tamanho da amostra foi calculado a partir do número de idosos no município em 2007 (7\% de 1.249.645 habitantes), considerando os seguintes parâmetros: frequência esperada de $30 \%$ para os objetivos específicos do inquérito, intervalo de confiança de $95 \%$, precisão absoluta de $5 \%$ e efeito do desenho da amostra por conglomerados (DEFF) de 1,8. Acrescentando-se $11 \%$ para perdas, a amostra foi estimada em 934 idosos, suficiente para estudar a autoavaliação de saúde em idosos de Goiânia, tendo em vista que sua prevalência foi de $27 \%$ em estudo anterior no município' ${ }^{1}$ parâmetro inferior ao considerado na amostra maior para prevalência de desfechos.

Foram considerados critérios de inclusão: ter idade igual ou superior a 60 anos, residir na área urbana de Goiânia, residir e ter dormido mais de quatro noites por semana na residência. Foram excluídos os idosos que não responderam à pergunta sobre autoavaliação do estado de saúde.

Para selecionar os idosos, foi realizada amostragem por conglomerados. A área geográfica do estudo foi definida a partir de Setores Censitários (SC), identificando-se 1.068 SC no município, sendo 912 estritamente urbanos. A média de indivíduos por setor foi de 980 pessoas. Considerando-se 7\% de idosos na população de Goiânia (na época da coleta), foram estimados 16,3 idosos por SC. Dividindo-se o total da amostra $(n=934)$ pelo número de idosos estimados 
por SC (17), seriam necessários 55 SC para a coleta dos dados. Foi sorteado um setor a mais (56) por meio de tabela de números aleatórios, criada em sistema eletrônico de randomização. Nesses setores, foram sorteados o quarteirão e a esquina para o início da coleta. A partir da esquina sorteada, a primeira residência foi visitada, e, caso não houvesse idoso, o pesquisador de campo se deslocava para o próximo domicílio até identificar um idoso. Na ocorrência de mais de um idoso residindo no domicílio, todos eram entrevistados.

Foi realizado estudo-piloto com 50 idosos residentes em um SC não sorteado para a coleta dos dados, cujo objetivo foi qualificar a equipe e refinar os instrumentos de coleta de dados. Após o estudo-piloto, os dados foram coletados entre dezembro de 2009 e 2010 por pesquisadores de campo previamente treinados. Os questionários foram preenchidos durante a entrevista, posteriormente codificados e entregues em uma secretaria central para checagem de inconsistências. Mediante inconsistências, o entrevistador retornava ao domicílio e corrigia os registros antes da digitação no banco de dados.

A variável desfecho foi a autoavaliação negativa de saúde, avaliada por meio da resposta à seguinte pergunta: "Em geral, o(a) Sr.(a) diria que sua saúde é?". As opções de respostas eram: muito boa, boa, regular, ruim e muito ruim. A variável foi dicotomizada em autoavaliação de saúde boa (muito boa/boa/regular) e ruim (ruim/muito ruim) ${ }^{5,18}$

As variáveis de exposição foram demográficas (sexo, faixa etária, renda e escolaridade), condições de saúde (número de doenças, quedas no último ano e internações no último ano) e capacidade funcional. As doenças foram autorreferidas pelo idoso, por meio da resposta à pergunta: "Quais doenças o médico já disse que o Sr.(a) tem?". As doenças relatadas foram enumeradas para identificação do número de doenças. A capacidade funcional foi avaliada por meio de AVDs, identificadas como ABVDs e AIVDs. O comprometimento nas AVDs foi identificado por meio da escala de Katz ${ }^{19}$, que inclui a dependência e a independência do idosos para banhar-se, vestir-se, usar o banheiro, transferir-se e alimentar-se. Já as AIVDs foram avaliadas por meio da escala de Lawton ${ }^{20}$, que avalia a dependência e a independência para fazer compras, usar transporte, usar medicamentos, gerir as finanças, usar telefone, preparar refeições, arrumar a casa, fazer trabalhos manuais, lavar e passar roupa.

Os dados foram analisados no STATA 12.0. Todas as variáveis foram primeiramente analisadas de forma descritiva. O teste qui-quadrado de Pearson foi utilizado para comparar proporções. A magnitude da associação foi estimada por meio da razão de prevalência (RP), com variância robusta, e seus respectivos intervalos de confiança (IC95\%). Foi realizada análise múltipla hierarquizada, definindo-se três blocos de análises: bloco distal (sexo e faixa etária), bloco intermediário (número de doenças e internações) e bloco proximal (incapacidade para banhar-se, vestir-se, usar o banheiro, transferir-se, alimentar-se, fazer compras, usar medicamentos, gerir as finanças, usar transporte, usar telefone, preparar refeições, arrumar a casa, fazer trabalhos manuais, lavar e passar roupa). Foram incluídas no modelo múltiplo as variáveis que na análise bivariada apresentaram $p<0,20$.

\section{RESULTADOS}

Neste inquérito, foram entrevistados 934 idosos, dos quais 44 (4,7\%) foram excluídos por não terem respondido à questão referente à autoavaliação do estado de saúde. Portanto, a amostra final foi composta por 890 idosos.

Desse total, 62,4\% eram do sexo feminino, 48,1\% estavam na faixa etária de 60-69 anos, $57,9 \%$ possuíam renda inferior a um salário mínimo e $48,2 \%$ tinham nível de escolaridade na fase do ensino primário. Quanto às condições de saúde, $66,5 \%$ possuíam três ou mais doenças autorreferidas e $22,1 \%$ foram internados no último ano (Tabela 1).

A distribuição da autoavaliação da saúde foi: $11,9 \%$ muito boa, 32,7\% boa, 43,9\% regular, 8,9\% ruim e $2,6 \%$ muito ruim. A prevalência global de autoavaliação negativa foi de $11,5 \%(n=102)$.

$\mathrm{Na}$ análise bivariada, foram associadas à autoavaliação de saúde ruim: sexo feminino $(R P=1,59$; IC95\% 1,05-2,41), idade superior a 80 anos ( $R P=1,69$; IC95\% 1,08-2,66), presença de três ou mais doenças $(\mathrm{RP}=6,83$; IC95\% 3,21-14,5) e internação no último ano $(\mathrm{RP}=2,39$; IC95\% 1,63; 3,50) (Tabela 1). 
A prevalência de ABVDs foi de aproximadamente $6 \%$, sendo que, para as atividades vestir-se e alimentar-se, os idosos apresentaram maior dependência. Quanto às AIVDs, as mais prevalentes foram usar transporte $(44,7 \%)$ e usar telefone (27,8\%). Tanto as ABVDs quanto as AIVDs apresentaram associação estatisticamente significante com a percepção de saúde negativa na análise bivariada $(p<0,05)$ (Tabela 2 e 3).

No modelo final de análise múltipla, permaneceram associadas à autoavaliação de saúde ruim: número de morbidades, internações, incapacidade para transferir-se, fazer compras, usar transporte e usar telefone (Tabela 4).

Tabela 1. Prevalência de autoavaliação de saúde ruim e associação com variáveis demográficas e de saúde em idosos residentes. Goiânia, Goiás, Brasil, $2010(n=890)$

\begin{tabular}{|c|c|c|c|c|c|}
\hline \multirow{2}{*}{ Variáveis } & \multirow{2}{*}{ n (\%) } & \multicolumn{2}{|c|}{ Prevalência } & \multirow{2}{*}{ RP (IC95\%) } & \multirow{2}{*}{$p<0,05$} \\
\hline & & Boa n (\%) & Ruim n (\%) & & \\
\hline Sexo & & & & & 0,027 \\
\hline Feminino & $555(62,4)$ & $481(86,7)$ & $74(13,3)$ & $1,59(1,05-2,41)$ & \\
\hline Masculino & $335(37,6)$ & $307(91,6)$ & $28(8,4)$ & 1,00 & \\
\hline Faixa etária & & & & & 0,060 \\
\hline 60-69 anos & $428(48,1)$ & $386(90,2)$ & $42(9,8)$ & 1,00 & \\
\hline 70-79 anos & $300(33,7)$ & $267(89,0)$ & $33(11,0)$ & $1,12(0,72-1,72)$ & \\
\hline$\geq 80$ anos & $162(18,2)$ & $135(83,4)$ & $27(16,6)$ & $1,69(1,08-2,66)$ & \\
\hline Número de doenças & & & & & $<0,001^{\S}$ \\
\hline $0-2$ & $298(33,5)$ & $291(97,7)$ & $7(2,3)$ & 1,00 & \\
\hline 3 ou mais & $592(66,5)$ & $497(83,9)$ & $95(16,0)$ & $6,83(3,21-14,5)$ & \\
\hline Internação & & & & & $<0,001$ \\
\hline Sim & $197(24,3)$ & $157(79,7)$ & $40(20,3)$ & $2,39(1,63-3,50)$ & \\
\hline Não & $614(75,7)$ & $562(91,6)$ & $52(8,4)$ & 1,00 & \\
\hline
\end{tabular}

RP: razão de prevalência; IC: intervalo de confiança; ${ }^{5}$ Teste exato de Fisher

Tabela 2. Análise univariada bruta entre autoavaliação de saúde negativa e dependência para as atividades básicas de vida diária (ABVDs). Goiânia, Goiás, Brasil, 2010 ( $n=890)$

\begin{tabular}{|c|c|c|c|c|c|}
\hline \multirow{2}{*}{ Variáveis } & \multirow{2}{*}{ n (\%) } & \multicolumn{2}{|c|}{ Prevalência } & \multirow{2}{*}{ RP (IC95\%) } & \multirow{2}{*}{$\boldsymbol{p}$} \\
\hline & & Boa n (\%) & Ruim n (\%) & & \\
\hline Banhar-se & & & & & $<0,001$ \\
\hline Independente & $841(94,8)$ & $755(89,7)$ & $86(10,3)$ & 1,00 & \\
\hline Dependente & $46(5,2)$ & $31(67,4)$ & $15(32,6)$ & $3,18(2,01-5,05)$ & \\
\hline Vestir-se & & & & & $<0,001$ \\
\hline Independente & $831(93,7)$ & $746(89,7)$ & $85(10,3)$ & 1,00 & \\
\hline Dependente & $55(6,3)$ & $40(72,7)$ & $15(27,3)$ & $2,66(1,65-4,29)$ & \\
\hline Usar o banheiro & & & & & $<0,001$ \\
\hline Independente & $849(95,7)$ & $762(89,7)$ & $857(10,3)$ & 1,00 & \\
\hline Dependente & $38(4,3)$ & $24(63,2)$ & $14(36,8)$ & $3,59(2,26-5,70)$ & \\
\hline Transferir-se & & & & & $<0,001$ \\
\hline Independente & $849(95,7)$ & $765(90,2)$ & $84(9,8)$ & 1,00 & \\
\hline Dependente & $38(4,3)$ & $21(55,3)$ & $17(44,7)$ & $4,52(3,00-6,79)$ & \\
\hline Alimentar-se & & & & & $<0,001$ \\
\hline Independente & $836(94,3)$ & $765(90,0)$ & $85(10,0)$ & 1,00 & \\
\hline Dependente & $51(5,7)$ & $21(56,7)$ & $16(43,3)$ & $4,32(2,83-6,58)$ & \\
\hline
\end{tabular}

RP: razão de prevalência; IC: intervalo de confiança; $p=<0,05$ 
Tabela 3. Análise univariada bruta entre autoavaliação de saúde negativa e dependência para as atividades instrumentais de vida diária (AIVDs). Goiânia, Goiás, Brasil, 2010 ( $n=890)$

\begin{tabular}{|c|c|c|c|c|c|}
\hline \multirow{2}{*}{ Variáveis } & \multirow{2}{*}{ n (\%) } & \multicolumn{2}{|c|}{ Prevalência } & \multirow{2}{*}{ RP (IC95\%) } & \multirow{2}{*}{$p$} \\
\hline & & Boa n (\%) & Ruim n (\%) & & \\
\hline Fazer compras & & & & & $<0,001$ \\
\hline Independente & $670(75,6)$ & $625(93,3)$ & $45(6,7)$ & 1,00 & \\
\hline Dependente & $216(24,4)$ & $160(74,1)$ & $56(25,9)$ & $3,86(2,68-5,54)$ & \\
\hline Usar transporte & & & & & $<0,001$ \\
\hline Independente & $849(95,7)$ & $765(90,2)$ & $84(9,8)$ & 1,00 & \\
\hline Dependente & $38(4,3)$ & $21(55,3)$ & $17(44,7)$ & $4,52(3,00-6,79)$ & \\
\hline Usar medicamentos & & & & & $<0,001$ \\
\hline Independente & $762(86,1)$ & $696(91,4)$ & $66(8,6)$ & 1,00 & \\
\hline Dependente & $123(13,9)$ & $88(71,6)$ & $35(28,4)$ & $3,28(2,28-4,72)$ & \\
\hline Gerir as finanças & & & & & $<0,001$ \\
\hline Independente & $741(83,6)$ & $675(91,1)$ & $66(8,9)$ & 1,00 & \\
\hline Dependente & $145(16,4)$ & $110(75,8)$ & $35(24,2)$ & $2,71(1,87-3,92)$ & \\
\hline Usar telefone & & & & & $<0,001$ \\
\hline Independente & $770(83,1)$ & $678(91,9)$ & $60(8,1)$ & 1,00 & \\
\hline Dependente & $157(16,9)$ & $106(72,1)$ & $41(27,9)$ & $3,43(2,40-4,90)$ & \\
\hline Preparar refeições & & & & & $<0,001$ \\
\hline Independente & $754(81,4)$ & $662(61,2)$ & $64(8,8)$ & 1,00 & \\
\hline Dependente & $172(18,6)$ & $122(76,7)$ & $37(23,3)$ & $2,63(1,82-3,80)$ & \\
\hline Arrumar a casa & & & & & $<0,001$ \\
\hline Independente & $600(64,7)$ & $531(92,7)$ & $42(7,3)$ & 1,00 & \\
\hline Dependente & $327(35,3)$ & $254(81,1)$ & $59(18,8)$ & $2,57(1,77-3,72)$ & \\
\hline Fazer trabalhos manuais & & & & & $<0,001$ \\
\hline Independente & $534(57,6)$ & $475(94,0)$ & $30(5,9)$ & 1,00 & \\
\hline Dependente & $393(42,4)$ & $310(81,4)$ & $71(18,6)$ & $3,13(2,09-4,70)$ & \\
\hline Lavar e passar roupa & & & & & $<0,001$ \\
\hline Independente & $549(59,3)$ & $493(93,9)$ & $32(6,1)$ & 1,00 & \\
\hline Dependente & $377(40,7)$ & $291(80,8)$ & $69(19,2)$ & $3,14(2,11-4,67)$ & \\
\hline
\end{tabular}

RP: razão de prevalência; IC: intervalo de confiança; $p=<0,05$

Tabela 4. Análise múltipla hierarquizada entre autoavaliação de saúde negativa e variáveis demográficas, condições de saúde e dependência para as atividades básicas de vida diária (ABVDs) e atividades instrumentais de vida diária (AIVDs). Goiânia, Goiás, Brasil, 2010 ( $n=890)$

\begin{tabular}{|c|c|c|c|c|c|c|}
\hline \multirow{2}{*}{ Variáveis } & \multicolumn{2}{|c|}{ Bloco distal } & \multicolumn{2}{|c|}{ Bloco intermediário } & \multicolumn{2}{|c|}{ Bloco proximal } \\
\hline & RPaj' (IC95\%) & $p$ & $\mathrm{RPaj}^{2}(\mathrm{IC} 95 \%)$ & $p$ & $\mathrm{RPaj}^{3}$ (IC95\%) & $p<0,05$ \\
\hline \multicolumn{7}{|l|}{ Sexo } \\
\hline Masculino & 1,00 & & 1,00 & & 1,00 & \\
\hline Feminino & $1,60(1,06-2,42)$ & 0,024 & $1,30(0,84-2,00)$ & 0,231 & $1,45(0,94-2,24)$ & 0,091 \\
\hline \multicolumn{7}{|c|}{ Faixa etária (anos) } \\
\hline $60-69$ & 1,00 & & 1,00 & & 1,00 & \\
\hline 70-79 & $1,13(0,73-1,74)$ & 0,573 & $0,90(0,58-1,41)$ & 0,669 & $0,63(0,40-0,99)$ & 0,046 \\
\hline$\geq 80$ & $1,71(1,09-2,68)$ & 0,018 & $1,36(0,87-2,13)$ & 0,171 & $0,73(0,43-1,22)$ & 0,235 \\
\hline
\end{tabular}

IC: intervalo de confiança; $\mathrm{O}$ uso de transporte não permaneceu no modelo de análise múltipla por ter apresentado colinearidade com a ABVD transferir-se; RPaj': razão de prevalência ajustada por sexo e idade; RPaj': razão de prevalência ajustada por sexo, faixa etária, número de doenças e internações; RPaj ${ }^{3}$ : razão de prevalência ajustada por sexo, faixa etária, número de doenças, internações, incapacidade para banhar-se, vestir-se, ir ao banheiro, transferir-se, alimentar-se, fazer compras, usar medicamentos, gerir as finanças, usar transporte, usar telefone, preparar refeições, arrumar a casa, fazer trabalhos manuais, lavar e passar roupa 
Tabela 4. Continuação...

\begin{tabular}{|c|c|c|c|c|c|c|}
\hline \multirow{2}{*}{ Variáveis } & \multicolumn{2}{|c|}{ Bloco distal } & \multicolumn{2}{|c|}{ Bloco intermediário } & \multicolumn{2}{|c|}{ Bloco proximal } \\
\hline & RPaj' $^{1}$ (IC95\%) & $p$ & $\mathrm{RPaj}^{2}$ (IC95\%) & $p$ & $\mathrm{RPaj}^{3}$ (IC95\%) & $p<0,05$ \\
\hline \multicolumn{7}{|l|}{ Número de doenças } \\
\hline $0-2$ & - & & 1,00 & & 1,00 & \\
\hline 3 ou mais & - & & $5,81(2,59-13,04)$ & $<0,001$ & $4,26(1,89-9,62)$ & $<0,001$ \\
\hline \multicolumn{7}{|l|}{ Internação (sim) } \\
\hline Não & - & & 1,00 & & 1,00 & \\
\hline Sim & - & & $2,08(1,43-3,04)$ & $<0,001$ & $1,59(1,10-2,28)$ & 0,012 \\
\hline \multicolumn{7}{|l|}{ Incapacidade para: } \\
\hline Banhar-se & - & & - & & $0,41(0,78-2,20)$ & 0,303 \\
\hline Vestir-se & - & & - & & $0,34(0,05-2,15)$ & 0,255 \\
\hline Ir ao banheiro & - & & - & & $1,35(0,35-5,20)$ & 0,662 \\
\hline Transferir-se & - & & - & & $2,60(1,30-8,45)$ & 0,020 \\
\hline Alimentar-se & - & & - & & $1,93(0,91-4,07)$ & 0,082 \\
\hline Fazer compras & - & & - & & $1,98(1,16-3,39)$ & 0,012 \\
\hline Usar medicamentos & - & & - & & $1,61(0,79-3,30)$ & 0,188 \\
\hline Gerir as finanças & - & & - & & $0,62(0,30-1,26)$ & 0,188 \\
\hline Usar transporte & - & & - & & $3,86(1,41-10,51)$ & 0,008 \\
\hline Usar telefone & - & & - & & $1,60(1,00-2,63)$ & 0,050 \\
\hline Preparar refeições & - & & - & & $0,98(0,53-1,79)$ & 0,949 \\
\hline Arrumar a casa & - & & - & & $1,04(0,61-1,76)$ & 0,877 \\
\hline $\begin{array}{l}\text { Fazer trabalhos } \\
\text { manuais }\end{array}$ & - & & - & & $1,06(0,59-1,91)$ & 0,830 \\
\hline Lavar e passar roupa & - & & - & & $1,75(0,95-3,20)$ & 0,068 \\
\hline
\end{tabular}

IC: intervalo de confiança; O uso de transporte não permaneceu no modelo de análise múltipla por ter apresentado colinearidade com a ABVD transferir-se; RPaj': razão de prevalência ajustada por sexo e idade; $\mathrm{RPaj}^{2}$ : razão de prevalência ajustada por sexo, faixa etária, número de doenças e internações; RPaj ${ }^{3}$ : razão de prevalência ajustada por sexo, faixa etária, número de doenças, internações, incapacidade para banhar-se, vestir-se, ir ao banheiro, transferir-se, alimentar-se, fazer compras, usar medicamentos, gerir as finanças, usar transporte, usar telefone, preparar refeições, arrumar a casa, fazer trabalhos manuais, lavar e passar roupa

\section{DISCUSSÃO}

Os resultados deste estudo demonstram que a dependência para as atividades de transferir-se, fazer compras, usar transporte e usar telefone teve associação independente com a autoavaliação negativa de saúde, reforçando a importância de incluí-las na avaliação de idosos em diferentes contextos da atenção à saúde, uma vez que a dependência pode refletir em alterações nas condições reais de saúde e qualidade de vida dos idosos. Esses resultados são importantes para a prática clínica, pois apontam a necessidade de vigilância e acompanhamento das AVDs como reflexo da saúde dos idosos.

A prevalência geral de autoavaliação de saúde ruim em idosos foi de $11,4 \%(n=102)$, sendo semelhante a um estudo realizado no Japão $(11,1 \%)^{21}$, porém inferior ao encontrado em uma ampla revisão sistemática com estudos nacionais, que apontou variação de $51,9 \%$ a 12,6\%5. Estudos dos últimos cinco anos com idosos têm apresentado diferentes prevalências: $15 \%$ na Paraíba ${ }^{7}, 18 \%$ em São Paulo ${ }^{22}$ e $30 \%$ em Minas Gerais (30\%) ${ }^{3}$. Os estudos mostram que as variações na distribuição da autoavaliação da saúde entre os estados brasileiros permaneceram as mesmas nos estudos mais atuais ${ }^{3,7,18}$, o que reforça o uso desse indicador para acompanhamento das condições de saúde de idosos. Uma análise longitudinal das desigualdades sociais e autoavaliação de saúde, utilizando dados do estudo Saúde Bem-estar e Envelhecimento $(\mathrm{SABE})^{18}$, aponta que piores indicadores de renda, escolaridade, classes de consumo, grupos etários e cor da pele são fatores que se associam diretamente e de forma persistente à autoavaliação ruim da saúde dos idosos. As evidências citadas ${ }^{3,5,7,22}$ demonstram que a persistência de iniquidades sociais entre os diferentes grupos sociais e as regiões 
brasileiras contribui para a pior percepção da saúde e consequentes desfechos negativos em saúde. Mais uma vez, deve ser reforçado o planejamento específico para promoção da saúde dos idosos, incluindo ações que visem à modificação da percepção de saúde deles com vistas à melhora da qualidade de vida.

Os estudos sobre autoavaliação de saúde mostram associação desse indicador com o aumento da idade ${ }^{1,3,5-7}$, o que é justificado pelo aumento de incapacidades e complicações com a saúde. Neste estudo, a faixa etária de 70 a 79 anos foi fator de proteção para a percepção negativa da saúde, o que precisa ser investigado em estudos futuros.

Em relação às condições de saúde analisadas, tanto o número de morbidades como as internações no último ano tiveram associação com a autopercepção negativa da saúde de forma independente. Essas evidências já foram bem exploradas na literatura ${ }^{1,3,5,6,13,14}$ e podem ser justificadas pelo fato de que em idosos as multimorbidades e a ocorrência de internações são fatores que afetam a dimensão biológica da saúde. Quanto maior o número de doenças, maior a demanda de cuidados e de prestação de serviços, o que, por consequência, pode implicar pior percepção de saúde no idoso.

A análise ajustada para todas as ABVDs e AIVDs revelou que, para as ABVDs, somente transferir-se manteve associação com a autoavaliação negativa da saúde. Já para as AIVDs, fazer compras, usar transporte e usar telefone manteve sua associação com a autoavaliação negativa da saúde.

De forma geral, a capacidade funcional expressa de forma global a saúde do idoso, ou seja, sua habilidade de executar o autocuidado, a necessidade de assistência e a capacidade de gerir sua própria vida9,12. Assim, a perda da dependência em ABVDs ou AIVDs apresenta papel central em como o idoso percebe a sua saúde.

Sendo assim, em relação aos tipos de ABVDs, transferir-se foi a única que manteve associação após a análise ajustada. Trata-se de uma atividade que requer integridade dos sistemas articular e musculoesquelético, além da manutenção do equilíbrio corporal ${ }^{23}$. Assim, o comprometimento nessa atividade pode sinalizar perda de mobilidade e dificuldade em executar outras ações cotidianas, que estão implicadas com o autocuidado dos idosos. Com as limitações presentes, o idoso diminui a interação e o contato com as pessoas ${ }^{24}$, desfavorecendo, assim, sua interação social e promovendo seu isolamento, podendo aumentar sua percepção de uma saúde ruim 25,26 .

Quanto às AIVDs, a dependência para fazer compras, cuidar das finanças e usar telefone manteve sua associação com a autoavaliação de saúde ruim. Estudo com dados da Pesquisa Nacional de Saúde (2013) $)^{27}$ mostrou que as chances de idosos com limitações em AIVDs relatarem percepção de saúde ruim aumentaram em 2,04 vezes. As AIVDs são tarefas de maior complexidade que as ABVDs e exigem, além das habilidades motoras, a capacidade cognitiva para sua execução ${ }^{28}$. Diante da privação de autonomia e da consequente necessidade de auxílio de tarefas que envolvem sua decisão, sua percepção de saúde pode ser afetada, o que o leva a classificá-la como ruim 29,30 .

Em relação às limitações deste estudo, destaca-se a impossibilidade de inferência de causalidade entre a autoavaliação de saúde ruim e as variáveis de exposição, por se tratar de um estudo transversal. Destaca-se também que a estratificação por tipo de incapacidade pode diminuir a precisão dos resultados. No entanto, trata-se de uma amostra representativa de uma capital brasileira, agregando novos resultados aos fatores específicos que podem se relacionar à percepção negativa da saúde.

A prevalência de autopercepção negativa do estado de saúde encontrada é ligeiramente inferior à média nacional. Porém, a associação com variáveis como número de doenças, internações, fazer compras, cuidar das finanças e usar telefone sugere a necessidade de vigilância e acompanhamento pelo serviço de saúde. Tendo em vista que a funcionalidade é um paradigma do envelhecimento e sinaliza alterações em diferentes dimensões da saúde dos idosos, acompanhar as perdas funcionais é imprescindível para o diagnóstico precoce de agravos nos idosos.

A Linha de Cuidado para Atenção Integral à Saúde da Pessoa prevê itinerários do usuário pela Rede de Atenção à Saúde, de acordo com as suas necessidades de saúde e sua capacidade 
funcional ${ }^{31}$. Nesse sentido, reitera-se a importância de ações de promoção da saúde para os idosos com comprometimento em diferentes tipos de AVDs, com o intuito de melhorar suas condições gerais de saúde.

O estudo inova ao retratar detalhadamente a relação de dependência/independência para as atividades cotidianas com a autoavaliação ruim de saúde, permitindo, assim, uma análise de quais atividades implicam maior comprometimento para a autonomia e independência dos idosos. Sugere-se que estudos analisem intervenções para melhora da capacidade funcional de idosos e seu impacto na percepção sobre a saúde.

\section{REFERÊNCIAS}

1. Pagotto V, Nakatani AYK, Silveira EA. Fatores associados e autoavaliação de saúde ruim em idosos usuários do Sistema Único de Saúde. Cad Saude Publica. 2011;27(8):1593-602. http://dx.doi.org/10.1590/S0102311X2011000800014. PMid:21877007.

2. Borim FSA, Barros MBA, Neri AL. Autoavaliação da saúde em idosos: pesquisa de base populacional no Municipio de Campinas, São Paulo, Brasil. Cad Saude Publica. 2012;28(4):769-80. http://dx.doi.org/10.1590/ S0102-311X2012000400016. PMid:22488322.

3. Santos EC, Couto BDM, Bastone ADC. Fatores associados à autoavaliação negativa da saúde em idosos cadastrados nas unidades básicas de saúde. ABCS Health Sci. 2018;43(1):47-54. http://dx.doi.org/10.7322/ abcshs.v43i1.999.

4. Lima-Costa MF, Cesar CC, Chor D, Proietti FA. Self-rated health compared with objectively measured health status as a tool for mortality risk screening in older adults: 10-year follow-up of the Bambuí Cohort Study of Aging. Am J Epidemiol. 2012;175(3):228-35. http://dx.doi.org/10.1093/aje/kwr290. PMid:22193172.

5. Pagotto V, Bachion MM, Silveira EA. Autoavaliação da saúde por idosos brasileiros: revisão sistemática da literatura. Rev Panam Salud Publica. 2013;33(4):302-10. http://dx.doi.org/10.1590/S102049892013000400010. PMid:23698180.

6. Loyola Al Fo, Firmo JOA, Uchoa E, Lima-Costa MF. Fatores associados à autoavaliação negativa da saúde entre idosos hipertensos e/ou diabéticos: resultados do projeto Bambuí. Rev Bras Epidemiol. 2013;16(3):559-71. http://dx.doi.org/10.1590/S1415-790X2013000300001. PMid:24896270.

7. Belém PLO, Melo RLP, Pedraza DF, Menezes TN. Autoavaliação do estado de saúde e fatores associados em idosos cadastrados na Estratégia Saúde da Família de Campina Grande, Paraíba. Rev Bras Geriatr Gerontol. 2016;19(2):265-76. http://dx.doi.org/10.1590/1809-98232016019.140206.

8. Nunes JD, Saes MO, Nunes BP, Siqueira FCV, Soares DC, Fassa ME, et al. Indicadores de incapacidade funcional e fatores associados em idosos: estudo de base populacional em Bajé, Rio Grande do Sul. Epidemiol Serv Saude. 2017;26(2):295-304. http://dx.doi.org/10.5123/S1679-49742017000200007. PMid:28492771.

9. Giacomin KC, Duarte YAO, Camarano AA, Nunes DP, Fernandes D. Cuidados e limitações funcionais em atividades cotidianas - ELSI-Brasil. Rev Saude Publica. 2018;9s(52 Supl. 2):9s. http://dx.doi.org/10.11606/ S1518-8787.2018052000650. PMid:30379293.

10. Bleijenberg N, Zuithoff NPA, Smith AK, de Wit NJ, Schuurmans MJ. Disability in the individual ADL, IADL and Mobility among older adults: a prospective cohort study. J Nutr Health Aging. 2017;21(8):897-903. http://dx.doi.org/10.1007/s12603-017-0891-6. PMid:28972242.

11. Zhang H, Wang ZH, Wang LM, Qi SG, Li ZX. Study on activities of daily living disability in communitydwelling older adults in China. Rev Chinesa de Epidemio. 2019;40(3):266-71. PMid:30884602.

12. Brito KQD, Menezes TN, Olinda RA. Incapacidade funcional: condições de saúde e prática de atividade física em idosos. Rev Bras Enferm. 2016;69(5):825-32. http://dx.doi.org/10.1590/0034-7167.2016690502. PMid:27783723.

13. Simonsson B, Molarius A. Self-rated health and associated factors among the oldest-old: results from a cross-sectional study in Sweden. Arch Public Health. 2020;78(1):6. http://dx.doi.org/10.1186/s13690-0200389-2. PMid:32025299.

14. Tomioka K, Kurumatani N, Hosoj H. Self-ratedpredicts in instrumental activiesofdaily living among highfunctioningcommunity-dwelling older people. Age Ageing. 2017;46(2):265-70. PMid:27614076.

15. Hirosaki M, Okumiya $K$, Wada T, Ishine M, Sakamoto R, Ishimoto $Y$, et al. Self-rated health is associated with subsequente functional decline among older adults in Japan. Int Psychogeriatr. 2017;29(9):1475-83. http://dx.doi.org/10.1017/S1041610217000692. PMid:28560936. 
16. Instituto Brasileiro de Geografia e Estatística. Censo demográfico 2018 [Internet]. Rio de Janeiro: IBGE; 2018 [citado em 2019 out 2]. Disponível em: https://www.ibge.gov.br/cidades-e-estados/go/goiania.html?

17. Santos TRA, Silveira EA, Pereira LV, Provin MP, Lima DM, Amaral RG. Potential drug-drug interactions in older adults: a population-based study. Geriatr Gerontol Int. 2017;17(12):2336-46. http://dx.doi.org/10.1111/ ggi.13070. PMid:28635169.

18. Antunes JLF, Chiavegatto FADP, Duarte YAO, Lebrão ML. Desigualdades sociais na autoavaliação de saúde dos idosos da cidade de São Paulo. Rev Bras Epidemiol. 2018;21(21 Supl. 2):1800-10. http://dx.doi. org/10.1590/1980-549720180010.supl.2. PMid:30726355.

19. Katz S, Akpom CAA. measure of primary sociobiological functions. Int J Health Serv. 1976;6(3):493-508. http://dx.doi.org/10.2190/UURL-2RYU-WRYD-EY3K. PMid:133997.

20. Lawton MP, Brody EM. Assessment of older people: self maintaining and instrumental activities of daily living. Gerontologist. 1969;9(3):179-86. http://dx.doi.org/10.1093/geront/9.3_Part_1.179.PMid:5349366.

21. Yoshimitsu K, Tabira T, Kubota M, Ikeda loue K, Akasaki Y. Factors afecting the self-rated health of elderly individuals living alone: a cross-sectional study. BMC Res Notes. 2017;10(1):512. PMid:29073932.

22. Melo DM, Falsarella GR, Neri AL. Autoavaliação de saúde, envolvimento social e fragilidade em idosos ambulatoriais. Rev Bras Geriatr Gerontol. 2014;17(3):471-84. http://dx.doi.org/10.1590/18099823.2014.13172.

23. Sekaran NK, Choi H, Hayward RA, Langa KM. Fall associated difficulty with activies of daily living in functionally independente individuals aged 65 to 69 in the United States: a cohort study. J Am Geriatr Soc. 2013;61(1):96-100. http://dx.doi.org/10.1111/jgs.12071. PMid:23311555.

24. Geib LTC. Determinantes sociais da saúde do idoso. Cien Saude Colet. 2012;17(1):123-33. http://dx.doi org/10.1590/S1413-81232012000100015. PMid:22218546.

25. Cardoso JH, Costa JSD. Características epidemiológicas, capacidade funcional e fatores associados em idosos de um plano de saúde. Cien Saude Colet. 2010;15(6):2871-8. http://dx.doi.org/10.1590/S1413 81232010000600024. PMid:20922295.

26. Rocha JP, Oliveira GG, Neris JCD, Bós AMG, Bós AJG. Impact of clinical and socio-economic factors and self-perception of health on the functionality of the elderly. Geriatr Gerontol Aging. 2017;11(3):124-32. http://dx.doi.org/10.5327/Z2447-211520171700051.

27. Zanesco C, Bordin D, Santos CB, Müller EV, Fadel CB. Factors determining the negative perception of the health of Brazilian elderly people. Rev Bras Geriatr Gerontol. 2018;21(3):283-92. http://dx.doi. org/10.1590/1981-22562018021.170210.

28. De Vriendt P, Gorus E, Cornelis E, Velghe A, Petrovic M, Mets T. The process of decline in advanced activies of daily living: a qualitative explorative study in mild cognitive impairment. Int Psychogeriatr. 2012;24(6):97486. http://dx.doi.org/10.1017/S1041610211002766. PMid:22301014.

29. Belmonte JMMM, Pivetta NRS, Batistoni SST, Neri AL, Borim FSA. The association between self-rated health and functional capacity indicators. Geriatr Gerontol Aging. 2017;11(2):61-7. http://dx.doi.org/10.5327/ Z2447-211520171700021.

30. Freitas MC, Queiroz AT, Sousa JAV. O significado da velhice e da experiência de envelhecer para os idosos. Rev Esc Enferm USP. 2010;44(2):407-12. http://dx.doi.org/10.1590/S0080-62342010000200024. PMid:20642054.

31. Brasil. Ministério da Saúde. Secretaria de Atenção à Saúde. Departamento de Ações Programáticas e Estratégicas. Orientações técnicas para a implementação de Linha de Cuidado para Atenção Integral à Saúde da Pessoa Idosa no Sistema Único de Saúde - SUS. Brasília: Ministério da Saúde; 2018. 91 p. 